\title{
TRANSFERENCIA DE ARSÉNICO EN EL SISTEMA AGUA- SUELO-MAÍZ DE Zea mays L. CULTIVADOS EN LA QUEBRADA DE CAMIÑA, NORTE DE CHILE
}

\author{
Venecia Herrera Apablaza ${ }^{1.23^{*}}$, Cristian Carrasco Farías ${ }^{1.2}$, Patricia Sandoval Cárcamo ${ }^{2}$, \\ Claudia Cortés Carvajal²
}

\begin{abstract}
RESUMEN
En la quebrada de Camiña, norte de Chile, las aguas naturales usadas para cultivar maíz (Zea mays L.), sobrepasan la concentración de arsénico en cinco veces la legislación chilena NCh1333. Se determinó su capacidad de fitoextracción del elemento, por el factor de bioconcentración (FBC) y si hay riesgo en el consumo de granos y hojas, mediante el cálculo del porcentaje del factor de translocación (PFT) en plantas adultas, obtenidas de seis predios del valle de Camiña, integrando la caracterización de aguas y suelos. Las concentraciones en agua alcanzaron $0,41 \pm 0,09 \mathrm{mg} / \mathrm{L}$; los suelos contaminados presentaron diferencias significativas $(\mathrm{p}<0,05)$ entre los sitios, precisando tres niveles: 30,100 y $300 \mathrm{mg} / \mathrm{kg}$. Los valores en hojas y granos fue variable $3,9 \pm 2,0$ y $0,10 \pm 0,14 \mathrm{mg} / \mathrm{kg}$, respectivamente. No existe relación lineal entre suelo y raíz, ni con las partes aéreas. Las raíces absorben y acumulan arsénico, obteniendo FB de 2,36 $\pm 1,10(\mathrm{n}=30)$, sin embargo, no ocurre la transferencia de la raíz a la parte aérea, dado que los PFT obtenidos fueron bastante bajos (entre 1,57 a 5,90\% en hojas y 0,02 a $0,11 \%$ en granos), en consecuencia, no hay riesgo en su consumo.
\end{abstract}

Palabras clave: Arsénico, maíz, factor de biocencentración (FB), factor de translocación (FT), quebrada de Camiña

\section{TRANSFER OF ARSENIC IN THE WATER-SOIL-MAIZE SYSTEM OF Zea mays L. OF CULTIVATED IN THE QUEBRADA DE CAMI- NA, NORTHERN CHILE}

\begin{abstract}
In the Quebrada de Camiña, northern Chile, the natural waters used to grow maize (Zea mays L.), exceed the arsenic concentration five times the NCh1333. In this work, the capacity of phytoextraction by bioconcentration factor $(\mathrm{CBF})$ and the risk of grain and leaf consumption were determined by calculating percentages translocation factor (PFT) of leaf and grain in adult plants obtained from six sites of the valley of Camiña, integrating the characterization of waters and soils. The concentration in the water was $0.41 \pm 0.09 \mathrm{mg} / \mathrm{L}$. The contaminated

\footnotetext{
${ }^{1}$ Facultad de Ciencias de la Salud, Universidad Arturo Prat, Casilla 121, Iquique, Chile.

${ }^{2}$ Centro de Investigación en Medio Ambiente CENIMA, Universidad Arturo Prat, Casilla 121, Iquique, Chile.

${ }^{3}$ Centro de Investigación y Desarrollo en Recursos Hídricos CIDERH. Universidad Arturo Prat. Vivar 493, 3er

Piso. Casilla 121, Iquique. Chile. vherrera@unap.cl
} 
soils presented significant differences $(\mathrm{p}<0.05)$ between the sites, defining three levels: 30 , 100 and $300 \mathrm{mg} / \mathrm{kg}$. The values in leaves and grains were variable $3.9 \pm 2.0$ and $0.10 \pm 0.14$ $\mathrm{mg} / \mathrm{kg}$, respectively. There is no linear relationship between soil and root and aerial parts. The roots absorb and accumulate arsenic, obtaining CBF of $2.36 \pm 1.10(n=30)$, however, the transfer of the root to the aerial part does not occur, since the PFT obtained were quite low (between 1.57 to $5.90 \%$ in leaves and 0.02 to $0.11 \%$ in grains), consequently, there is no risk in consumption.

Key words: Arsenic, maize, biocontration factor (FB), translocation factor (FT), quebrada de Camiña

\section{INTRODUCCIÓN}

Arsénico y sus especies químicas son considerados como agentes cancerígenos para humanos y nocivos para animales y plantas. La mayor amenaza para la salud pública reside en el consumo de agua para beber, preparar alimentos y regar cultivos alimentarios por la transferencia en el sistema agua-suelo-cultivo. Las plantas tienen una enorme capacidad de acumular contaminantes desde el entorno y llevar a cabo su desintoxicación por diversos mecanismos, tales como la absorción por las raíces, su translocación y acumulación en tallos y hojas. La planta de maíz (Zea mays L.) posee gran valor nutritivo en almidón, proteínas, lípidos y otras biomoléculas, es el cereal de mayor cultivo en el planeta y posee características y habilidades de un fitorremediador ${ }^{1,2}$. Investigaciones en cultivos de maíz en Tanzania reportaron acumulación de arsénico en su raíz y muy bajos valores de porcentaje de factor de translocación en grano ${ }^{3}$. Baig et al. $(2010)^{1}$, demostraron que el orden en la capacidad de translocación de arsénico en planta de maíz fue mayor para la raíz que en tallo y en grano. En cultivos de la misma especie, cercanos a una área minera en México, se ha demostrado que depende de las características del suelo si ocurre la acumulación en la raíz y que prácticamente no ocurre translocación desde la raíz hacia diferentes partes de la planta, señalando que los contenidos en grano son siempre inferiores a aquellos obtenidos en hojas, tallo y raíz ${ }^{4}$. Existe un diagnóstico y se compararon, de la literatura disponible, las concentraciones y fitodisponibilidad de arsénico en diversos suelos agrícolas para cultivo de maíz, la transferencia desde el suelo hacia raíz-tallo y los porcentajes de factor de translocación desde la raíz hacia las partes aéreas (tallo-hojas-grano) ${ }^{5}$.

Es reconocido que en el medio ambiente del norte de Chile existen elevadas concentraciones de arsénico ${ }^{6,7}$. En suelos contaminados naturalmente con el elemento, de la precordillera de la región de Antofagasta se reportan valores de concentración para granos de maíz entre 0,02 a $0,40 \mathrm{mg} / \mathrm{kg}^{7}$. Por su parte, la región de Tarapacá posee un sistema hidrológico particular, las precipitaciones son escasas y ocurren en época estival sobre los 3500 metros sobre el nivel del mar (msnm). La única vertiente que desagua en el océano Pacífico es la quebrada de Camiña, cuyas aguas con 0,40 a $0,60 \mathrm{mg} / \mathrm{L}$ en arsénico están bajo incumplimiento de la legislación chilena, según $\mathrm{NCh} 1333^{8}$. Estas aguas del río y manantiales que se encuentran en las laderas de la quebrada son captadas para uso de riego en cultivos de maíz (Zea mays L.) 
en diversos predios y parcelas. Los choclos que se cultivan, llamados localmente choclos camiñanos, pertenecen a la variedad llamada Maíz Capio Chileno Chico o Maíz Blanco, de sabor dulce. En el norte grande de Chile, según el Instituto de Investigaciones Agropecuarias, se distribuye entre los 2600 a $3700 \mathrm{msnm}$ y es resistente al frío y las sequías. Las hojas de las plantas son usadas como fertilizantes y alimento para ganado familiar. Recientemente, la red de SERNAGEOMIN ha realizado un mapa de metales y metaloides en los suelos y sedimentos de la región de Tarapacá, donde se determinó la existencia de una rareza geoquímica de arsénico, denominada Anomalía Regional Putre - Camiña ${ }^{9}$. Aunando a esto, en esta zona, no existe información científica disponible relacionada con la transferencia de arsénico en los cultivos.

En este trabajo se determinó para arsénico total, la capacidad de fitoextracción de Zea mays L., mediante el factor de bioconcentración (FBC) en la raíz y el riesgo del consumo de grano y hojas, según valores del cálculo del porcentaje de factor de translocación (PFT) de plantas adultas obtenidas de seis predios del valle de Camiña, integrando la caracterización fisicoquímica de las matrices abióticas aguas de riego y suelos cultivados. También se consideró un sector agrícola lejano a la quebrada (Estación Experimental Canchones en la comuna de Pozo Almonte), como testigo.

\section{PARTE EXPERIMENTAL}

El área de estudio se sitúa al noreste de la ciudad de Iquique, en la Precordillera (valle) de la quebrada de Camiña (19 $12^{\prime}-19^{\circ} 32 \mathrm{~S}$ y $\left.69^{\circ} 45^{\prime}-69^{\circ} 12 \mathrm{~W}\right)$, norte de Chile. La quebrada es una unidad hidrológica superficial del tipo cuenca exorreica que desemboca en la localidad de Pisagua. Posee longitud de $140 \mathrm{~km}$ aproximadamente, desde su inicio en los alrededores del cerro Pumiri ubicado a una altura de $5484 \mathrm{msnm}$ La comuna de Camiña posee 19 localidades con aproximadamente 1100 habitantes que viven en caseríos ubicados en terrazas aluviales. La principal actividad económica es la agricultura destinada a la producción de hortalizas y verduras y en menor proporción la ganadería a nivel familiar. Considerando las características agroclimáticas de la comuna, existe una suerte de especialización de las zonas de cultivo para la producción de cierto tipo de especies, en la parte intermedia de la quebrada (localidades de Apamilca, Chapiquilta y Camiña) los cultivos que mejor se desarrollan son ajo blanco, cebolla, alfalfa y maíz.

Las campañas de terreno en el valle de Camiña se realizaron en octubre del año 2015. Se seleccionaron tres predios ubicados en la ladera sur del río y tres en la ladera norte. Se colectaron muestras de agua de riego (AR1-AR6) y a la vez, de suelo cultivado (S1- S6) los cuales presentaban cultivos de maíz, excepto del sector 2. De cada uno de estos sitios, se tomaron cuatro plantas de maíz (Zea mays L.), y se obtuvieron las muestras compuestas de las raíces (R1-R6), de las hojas (H1-H6) y de los granos (G1-G6). Muestras similares (testigos) fueron colectadas en suelos agrícolas lejanos a la quebrada y corresponden a la Estación Experimental de Canchones (EEC) de la Universidad Arturo Prat de Iquique, en la comuna de Pozo Almonte. En cada sitio se colectaron tres réplicas de las muestras de agua de riego, suelo y planta. 
En las muestras de agua se midieron la conductividad eléctrica (CE), el pH, el potencial redox y la concentración de oxígeno disuelto (OD) con equipo multiparamétrico WTW. Las muestras fueron filtradas a $0,45 \mu \mathrm{m}$ para analizar los sólidos totales disueltos (STD) mediante gravimetría a $105 \pm 5^{\circ} \mathrm{C}$; nitrato, $\mathrm{N}_{-N_{3}}$ por espectroscopía UV a $220 \mathrm{~nm}$; fosfato, $\mathrm{P}_{-} \mathrm{PO}^{4}$. ${ }_{3}$ por espectroscopía visible; boro, B por por el método de la azometina a $420 \mathrm{~nm}$, usando el equipo espectrofotómetro Arquimed Optizen UV/ Vis; y arsénico total, As, mediante espectroscopía de absorción atómica acoplado a generación de hidruro (EAA-GH) ${ }^{10}$.

Las muestras de suelo fueron secadas y tamizadas a $2 \mathrm{~mm}$ para determinar la humedad por gravimetría a $105 \pm 5^{\circ} \mathrm{C}$ por $48 \mathrm{~h}$ y se conservaron en desecadora. Se midieron el pH y la CE en extractos acuosos 1:2 (suelo:agua de calidad HPLC); se determinó la concentración de carbono orgánico facilmente oxidable (COFO) por valoración por retroceso con sulfato ferroso PA y la concentración de boro por azometina a $420 \mathrm{~nm}^{11}$. Para análisis de arsénico, se trabajó en la fracción inferior a $63 \mu \mathrm{m}$ de la muestra de suelo.

Las muestras compuestas de raíces, hojas y granos de choclo provenientes de las cuatro plantas de Zea mays L. se dejaron secar al aire por tres días en bandejas plásticas, teniendo precauciones para un ambiente libre de contaminantes. Los choclos blancos se desgranaron y los granos fueron lavados cuidadosamente con agua destilada, al igual que las hojas. Para liofilizar las muestras biológicas se congelaron por tres días a $-80^{\circ} \mathrm{C}$ en un congelador Gene Press Glacier $-86^{\circ} \mathrm{C}$ Ultralow Temperatura Freezer; luego fueron liofilizadas por 72 $\mathrm{h}$, en un equipo CHRIST modelo Alpha 1-4 LD plus y posteriormente pulverizadas con mortero de ágata, se guardaron en bolsas herméticas y se conservaron en desecadora, previa cuantificación de humedad.

La digestión del suelo se realizó con la mezcla de ácidos concentrados de calidad supra pure (sp) $\mathrm{HCl} / \mathrm{HNO}_{3} / \mathrm{HF} / \mathrm{H}_{3} \mathrm{BO}_{3}$ en bombas de teflón a alta presión a $170 \pm 5^{\circ} \mathrm{C}$ por $3 \mathrm{~h}^{12}$. Se realizaron siete pruebas para seleccionar la mejor digestión de las matrices bióticas seleccionando para hojas y grano, la mezcla de ácidos concentrados de calidad supra pure (sp) $\mathrm{HNO}_{3} / \mathrm{HCl} \mathrm{1:1} \mathrm{en} \mathrm{sistema} \mathrm{de} \mathrm{reflujo} \mathrm{a} 50{ }^{\circ} \mathrm{C}$ por $24 \mathrm{~h}$; para la raíz se utilizó la mezcla de ácidos concentrados de calidad supra pure (sp) $\mathrm{HNO}_{3} / \mathrm{HClO}_{4}$ 1:1 en sistema de reflujo a $80{ }^{\circ} \mathrm{C}$ por $24 \mathrm{~h}$. Los extractos se mantuvieron en envases de polietileno y refrigerados a $4{ }^{\circ} \mathrm{C}$. La determinación de arsénico total se llevó a cabo por EAA-GH, en un equipo Perkin Elmer modelo PinAAcle $900 \mathrm{H}$ con un generador de hidruros FIAS 100, previa pre-reducción de arsénico, desde As (V) a As (III), con KI en medio de ácido ascórbico y $\mathrm{HCl}$ concentrado. Para la curva de calibración entre 2,00 a 20,0 $\mu \mathrm{g} / \mathrm{L}$, se preparó desde una solución Tritrisol $1000 \mathrm{mg} / \mathrm{L}$ Merck. Se midió a 193,87 nm, en celda T de cuarzo a $900^{\circ} \mathrm{C}$, con llama de aireacetileno (20 y entre 70-80 psi, respectivamente) y argón (50-100 psi). El reactivo reductor usado fue $0,2 \% \mathrm{NaBH} 4$ en $0,3 \% \mathrm{NaOH}$ a $6 \mathrm{~mL} / \mathrm{min}$ y $\mathrm{HCl} 10 \%$ a flujo de $10 \mathrm{~mL} / \mathrm{min}$. Los análisis se hicieron en duplicado y la calidad de los resultados y validación de protocolos se evaluó mediante el análisis de material de referencia certificado "San Joaquín soil SRM 2709" y "Virginia Tobacco Leaves CTA-VTL-2" por duplicado, obteniéndose un 88 a 100\% de recuperación. Todas las concentraciones son expresadas en base a peso seco de la muestra. Se determinó el FBC por la proporción entre la concentración de arsénico en la raíz y en el suelo. Por su parte, se calculó el PFT para hojas y granos en relación al contenido en la raíz ${ }^{1,2,5}$. 


\section{RESULTADOS Y DISCUSIÓN}

La caracterización de los estadísticos básicos de parámetros de la calidad del agua de riego en el área de estudio y datos obtenidos del sector de la EEC, son resumidos en la tabla 1. Las mediciones de $\mathrm{pH}$ clasifican a las aguas como levemente alcalinas, sin mayores variaciones. La alcalinidad es producida por el equilibrio entre carbonatos y bicarbonatos, aunque en ocasiones contribuyen boratos y silicatos. El pH observado se encuentra en el intervalo de $\mathrm{pH}$ de las aguas naturales que se conserva entre 6,5 y 8,5 y es de relevancia en varios fenómenos fisicoquímicos y biológicos tales como reacciones redox, procesos de sorción y reacciones de solubilización-precipitación de iones en disolución ${ }^{10,14}$. Los valores de la concentración de OD establecen que son aguas oxigenadas, principalmente, por efecto de escorrentías, sin embargo, las mediciones del potencial redox indican un sistema acuático reductor. Los valores de CE y STD, relativamente altos para ríos, permiten establecer que son aguas dulces. Las concentraciones de nitrato y fosfato obtenidas son consideradas normales para aguas naturales, y la relación N/P proporcionó como resultado un amplio intervalo entre valores de 73 a 343, observándose que se encuentra muy por sobre la proporción para aguas continentales de $\mathrm{N} / \mathrm{P}=15$, que considera en el equilibrio, los procesos dinámicos de la fotosíntesis y reparación en el sistema acuático. El contenido de boro sobrepasa la máxima concentración admisible declarada en la legislación chilena, a través de la NCh 1333 para agua de riego que es $0,75 \mathrm{mg} / \mathrm{L}$ de $\mathrm{B}$.

Tabla 1. Estadísticos básicos de los parámetros físico-químicos de las muestras de agua de riego $(\mathrm{N}=24)$ de Camiña y valores para ECC (en unidades de $\mathrm{mg} / \mathrm{L}$ ).

\begin{tabular}{cccccccccc}
\hline & $\begin{array}{c}\mathbf{T} \\
\text { Estadístico }\end{array}$ & $\begin{array}{c}\mathbf{C E} \\
\left({ }^{\mathbf{0}} \mathbf{C}\right)\end{array}$ & $\begin{array}{c}\text { Redox } \\
(\boldsymbol{\mu S} / \mathbf{c m})\end{array}$ & $\mathbf{p H}$ & $\mathbf{O D})$ & $\mathbf{S T D}$ & $\mathbf{N}^{-N_{3}}{ }^{-}$ & $\mathbf{P}^{-P O_{4}}{ }^{-3}$ & $\mathbf{B}$ \\
\hline Min. & 13,8 & 933 & -86 & 8,16 & 5,29 & 529 & 9,9 & 0,02 & 7,8 \\
Max. & 15,8 & 1143 & -70 & 8,47 & 7,22 & 676 & 10,8 & 0,11 & 8,86 \\
Prom. & 15,3 & 1041 & -78 & 8,33 & 6,5 & 602 & 10,3 & 0,07 & 8,21 \\
DE & 0,7 & 78 & 5 & 0,09 & 0,65 & 44 & 0,2 & 0,03 & 0,34 \\
\hline ECC & 21,1 & 1290 & -15 & 8,07 & 5,49 & 936 & 10,03 & 0,07 & 4,91 \\
\hline
\end{tabular}

La figura 1 muestra, en diagrama de caja y bigote, la distribución de arsénico total en las aguas de riego usadas en los predios seleccionados del valle de Camiña y del sector de la EEC, observando que están bajo incumplimiento de la legislación chilena en Camiña, pero no aquellas del sector Canchones. Si se considera que la concentración máxima de arsénico permitida para el agua de riego según NCh.1333 es de $100 \mu \mathrm{g} / \mathrm{L}$, el valor promedio obtenido en este trabajo supera en cuatro a cinco veces la legislación $(0,41 \pm 0,09 \mathrm{mg} / \mathrm{L})$. Las aguas 
del sector 1 alcanzan el máximo valor. De manera similar las aguas usadas para el riego, en la cuenca del río Camarones, aledaña hacia el norte de la quebrada de Camiña, poseen elevadas concentraciones de arsénico ${ }^{6,9}$.

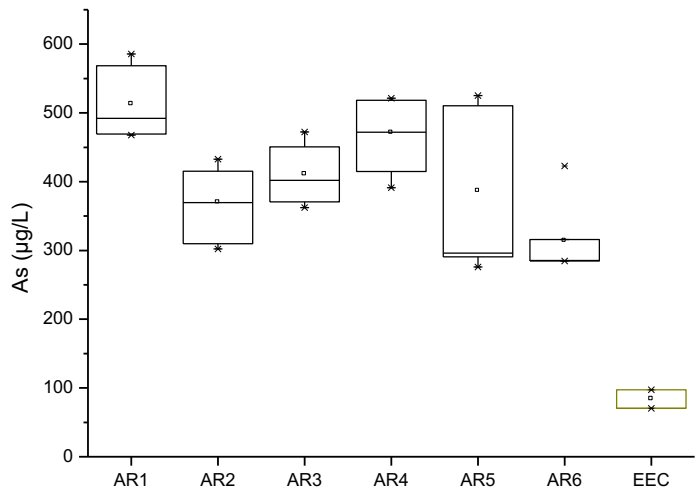

Figura 1. Distribución de arsénico en aguas de riego para los predios de Camiña y de la Estación Experimental Canchones (ECC).

La Organización Mundial de la Salud (OMS) ha sugerido una concentración máxima admisible en aguas de consumo humano de $10 \mu \mathrm{g} / \mathrm{L}$ y en Latinoamérica incluyendo Chile desde el año 2007 adoptó el nivel de $10 \mu \mathrm{g} / \mathrm{L}$, según la NCh 409, habiendo una excepción para la región de Arica y Parinacota y Tarapacá del $30 \mu \mathrm{g} / \mathrm{L}$ hasta el año 2017, en consecuencia, existe riesgo para la población rural expuesta, agricultores radicados y turistas que podrían llegar a consumir aguas naturales de la quebrada de Camiña.

Los resultados de la caracterización fisicoquímica de los suelos cultivados del valle de Camiña y del sector EEC se resumen en la tabla 2.

Tabla 2. Estadísticos básicos de los parámetros físicoquímicos de las muestras de suelos cultivados del valle de Camiña y valores del sector Canchones, EEC.

\begin{tabular}{cccccc}
\hline Estadístico & $\begin{array}{c}\mathbf{C E} \\
(\boldsymbol{\mu S} / \mathbf{c m})\end{array}$ & $\mathbf{p H}$ & $\begin{array}{c}\text { COFO } \\
\mathbf{( \% )}\end{array}$ & $\begin{array}{c}\mathbf{B} \\
(\mathbf{m g} / \mathbf{k g})\end{array}$ & $\begin{array}{c}\mathbf{A s} \\
(\mathbf{m g} / \mathbf{k g})\end{array}$ \\
\hline Mín. & 220 & 7,03 & 0,33 & 12,71 & 24,3 \\
Máx. & 911 & 8,22 & 3,73 & 33,74 & 375 \\
Prom. & 433 & 7,48 & 2,12 & 22,39 & 116 \\
DE & 217 & 0,34 & 1,21 & 5,23 & 94 \\
\hline EEC & $2394 \pm 35$ & $7,76 \pm 0,06$ & $0,45 \pm 0,05$ & $5,17 \pm 0,09$ & $50,1 \pm 2$ \\
\hline
\end{tabular}


La salinidad en los suelos, expresada por la CE, presenta un amplio intervalo siendo clasificados como suelos no salinos, por lo tanto no afecta a ningún cultivo, sin embargo, los suelos de la EEC son levemente salinos. La amplia variabilidad de los valores de CE, puede atribuirse a las condiciones climáticas propias de la zona, como: vientos que favorecen el transporte y decantación de sales desde la Pampa del Tamarugal, y los aluviones que ocurren en la quebrada durante la época estival de lluvias intensas en el Altiplano ${ }^{8}$. Además, el manejo de los suelos cultivados y el regadío por inundación, ya que parte de las aguas se infiltran en los suelos, mientras que el resto se evapora.

La medición del pH en un extracto acuoso del suelo permitió clasificarlos como suelos neutros a levemente alcalinos. Existen varios mecanismos que tamponan el $\mathrm{pH}$ de los suelos en los que participan, principalmente, hidróxido de aluminio, hidróxido de hierro, silicatos, dióxido de carbono, carbonatos y boratos entre otros. También influyen las reacciones de intercambio catiónico y los contenidos de sales en la superficie horizontal3,4. Los suelos en general presentan valores entre 4 y 8,5 unidades de $\mathrm{pH}$. Para suelos de zonas húmedas se considera normal entre 5 y 7 unidades de $\mathrm{pH}$, pero para suelos de regiones áridas los valores oscilan entre 7 y 9 unidades de $\mathrm{pH}^{6,12}$.

Las concentraciones de COFO clasifican a todos los suelos del tipo arenosos (COFO inferior al 5\%). Este tipo de suelo es habitual encontrarlos en zonas de clima árido y semiáridos ${ }^{7,12}$. La variabilidad se puede deber al manejo del suelo cultivado y/o la adición de fertilizantes de origen animal y vegetal. En general en el sector de S3, las muestras presentaron muy bajos valores de $\mathrm{COFO}$, en cambio en los sectores S2, S5 y S6, los contenidos fueron mayores. Como era de esperar, existe una correlación negativa entre el $\mathrm{pH}$ y el porcentaje de COFO presente en los suelos en estudio $\left(r^{2}=0,7083 ; y=2,9635 x+24,289\right)$.

El contenido total de boro en los suelos fue variable y elevado, en su mayoría no es asimilable por las plantas, el valor asimilable es por lo general del 5\%. En suelo arenoso, la concentración crítica con síntomas de carencia es de $0,3 \mathrm{mg} / \mathrm{kg}$ y el límite de concentración tóxica es de 5 $\mathrm{mg} / \mathrm{kg}^{15}$. Los rangos de concentración de boro en la solución del suelo que causa deficiencia o toxicidad en las plantas, son más pequeños que para cualquier otro elemento, sin embargo, dichos rangos son específicos para cada especie de planta, en consecuencia, es probable que los cultivos en la zona posean tolerancia a boro.

Los suelos cultivados del valle de Camiña presentaron elevados niveles de arsénico y diferencias significativas $(\mathrm{p}<0,05)$ entre los sitios y del sector Canchones, como se muestra en los diagrama de caja de la figura 3 . Niveles normales son entre $5-10 \mathrm{mg} / \mathrm{kg}$, siendo el promedio mundial de 7,2 mg/kg; mientras que en los suelos del continente americano es de $7,4 \mathrm{mg} / \mathrm{kg}$ y en suelos pantanosos de $13 \mathrm{mg} / \mathrm{kg}^{6,15}$. En la precordillera de la zona norte de Chile, los suelos están contaminados naturalmente; reportándose en suelos no cultivados entre $20-27 \mathrm{mg} / \mathrm{kg}$ y para suelos cultivados entre 64 y $448 \mathrm{mg} / \mathrm{kg} 6,8$. En Bangladesh la concentración de arsénico reportada está en un rango de 9 a $28 \mathrm{mg} / \mathrm{kg}$, en Alemania entre 2,5 a 4,1 mg/kg, en México de 2 a $40 \mathrm{mg} / \mathrm{kg}$ y en Argentina entre 0,8 a $22 \mathrm{mg} / \mathrm{kg}^{6}$. 


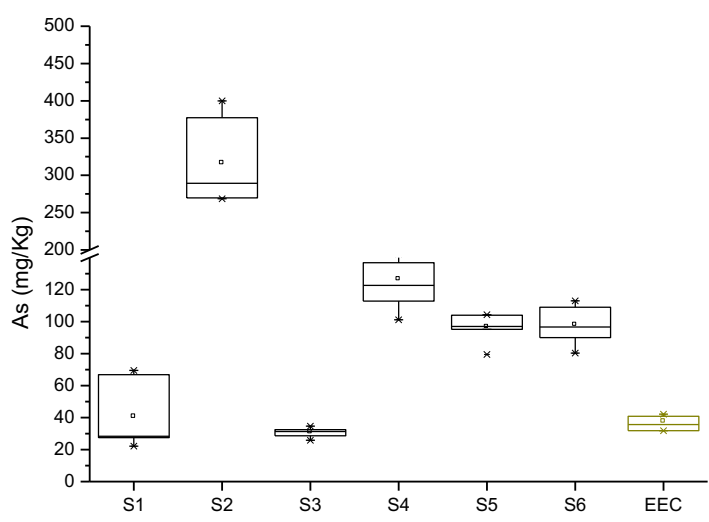

Figura 2. Distribución de arsénico en suelos cultivados del valle de Camiña y en el sector Canchones, ECC (testigo).

Los suelos del sector S2 presentaron el contenido más alto en arsénico, $304 \pm 51 \mathrm{mg} / \mathrm{kg}$. Les siguen los suelos de S4, S5 y S6 alcanzando un nivel similar de $101 \pm 7 \mathrm{mg} / \mathrm{kg}$. Esta concentración es análoga a la reportada en los suelos del área de la anomalía Putre - Camiña con $105 \pm 10 \mathrm{mg} / \mathrm{kg}^{9}$. La propagación en el diagrama de caja para el predio S1 se debe a que una de las réplicas presentó valores bajos de arsénico con un promedio de $26 \pm 2 \mathrm{mg} /$ $\mathrm{kg}$, y dos de estas presentaron el doble de esa concentración. Las muestras de la parcela S3 presentaron un promedio de $30,1 \pm 0,7 \mathrm{mg} / \mathrm{kg}$ y en sector de Canchomes $50 \mathrm{mg} / \mathrm{kg}$, lo que se puede considerar como un intervalo normal en suelos del norte de Chile. La variabilidad observada puede deberse a varios factores tales como: manejo de los suelos agrícolas, el método de fertilización utilizado, además de la frecuencia y abundancia del riego. El origen natural del arsénico en el suelo es el material parental del cual deriva y la adición, por las aguas de la cuenca ${ }^{6,8}$.

Para establecer relaciones entre la concentración de arsénico y los parámetros físico-químicos del suelo se omitió el sector S2. Existe una correlación negativa entre la concentración de arsénico y pH del suelo, cuyo coeficiente de correlación de Pearson $\left(\mathrm{r}^{2}=0,7339\right)$ confirma esta tendencia ya descrita por Rosas et al., (2014a) ${ }^{4}$. También se encontró una correlación lineal positiva entre arsénico y COFO $\left(\mathrm{r}^{2}=0,7449\right)$. El contenido de materia orgánica del suelo es un factor importante que puede influir en la disponibilidad y movilidad de arsénico ${ }^{6,12}$. Debido a la elevada variabilidad del arsénico en suelos, no existe correlación entre esta y las aguas usadas en el riego, lo que indica que la diferencia de arsénico y la adición del mismo en las aguas está asociada a un factor hidrogeológico y/o antropogénico de actividades agrícolas.

La concentración de arsénico en las raíces de la planta alcanzó un nivel bastante elevado con promedio de $146 \pm 45 \mathrm{mg} / \mathrm{kg}$ e importante variabilidad. La figura 3 muestra la distribución del metaloide en las raíces de las plantas, obtenidos de los seis predios y en el sector de Canchones, ECC. 


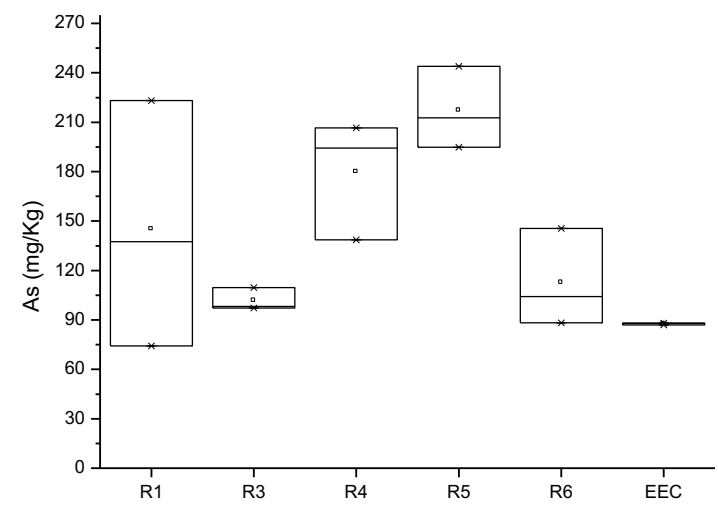

Figura 3. Distribución de arsénico en la raíz del maíz cultivado en los predios de Camiña y en el sector Canchones, ECC (testigo).

La mayor propagación de la serie de datos para las muestras compuestas del predio 1 (R1), se debe a que la raíz de la planta de una contramuestra presenta un valor elevado de 223 $\mathrm{mg} / \mathrm{kg}$ y en el mismo sector en otras contramuestras alcanzó un valor de $74 \mathrm{mg} / \mathrm{kg}$. Sin embargo, en el sector 3 y en sector EEC, las muestras presentan valores de arsénico en raíces con menos variabilidad y promedios similares. Dado que la mayoría de los suelos han sido catalogados como contaminados por arsénico, era de esperar que las raíces de estas plantas absorbieran y acumularan al elemento. Generalmente las plantas absorben las especies de arsénico que están disueltas en el suelo de cualquier forma iónica quelada o complejada, por los canales de fósforo, bioacumulándose en la raíz ${ }^{4,5,13}$. No se encontró correlación lineal entre la concentración de arsénico en el suelo y la raíz y, en consecuencia, los valores calculados de los FBC para los sitios seleccionados, la EEC y sus réplicas fueron variables (tabla 3 ). El valor promedio para el FBC en el área de estudio fue de 2,36 $\pm 1,10$ estableciendo la importante acumulación de arsénico en la raíz en todos los sectores estudiados, excepto en la parcela 4 en donde el FBC fue muy cercano a 1. Si se considera el sitio de referencia o testigo, EEC, su FBC cercano a 2 confirma la acumulación de arsénico en su raíz. Los resultados de FBC son comparables a los valores reportados en la literatura ${ }^{6}$ y aquellos valores determinados en los estudios reportados por Rosas et al. $(2014 a)^{5}$ con valores entre 0,04 a 8,0. El maíz cultivado en la zona posee habilidad para la fitorremediación de arsénico presente en el suelo.

Las concentraciones de arsénico obtenidas en las hojas de las plantas en estudio se muestran en los diagramas de caja de la figura 4. El valor promedio para Camiña fue de 3,9 $\pm 2,0$ $\mathrm{mg} / \mathrm{kg}$, la amplia variabilidad en los resultados se ve reflejado en la muestra compuesta $\mathrm{R} 3$, la cual reportó la concentración más baja de arsénico de $1,3 \mathrm{mg} / \mathrm{kg}$ y la muestra R1 la cual registró la mayor concentración de $8,9 \mathrm{mg} / \mathrm{kg}$. Según Kabata-Pendias, $(2011)^{15}$, la concentración suficiente o normal aproximada de arsénico en la hoja de tejido maduro para varias especies vegetales es de 1 a $1,7 \mathrm{mg} / \mathrm{kg}$ y el nivel crítico o tóxico se establece entre 5 a $20 \mathrm{mg} / \mathrm{kg}$. El 83\% de las muestras superan la concentración normal de arsénico en hojas y el $25 \%$ posee una concentración de arsénico crítico en sus hojas, por lo tanto, el consumo de hojas en ganado familiar puede resultar inadecuado 


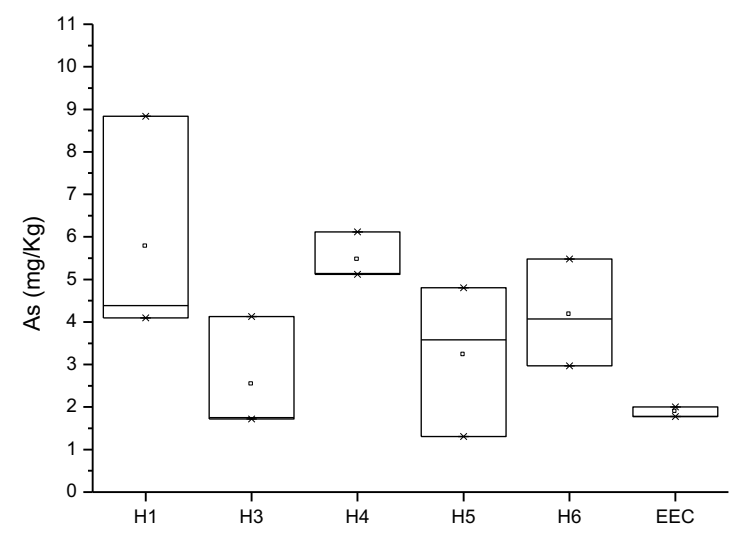

Figura 4. Distribución de arsénico en hojas de plantas de maíz, obtenidas de los seis predios del valle de Camiña.

Los resultados obtenidos en los granos del maíz muestran valores a nivel de ultratraza de la concentración de arsénico, tal como se muestra la distribución espacial en el área de estudio y el testigo en la figura 5.

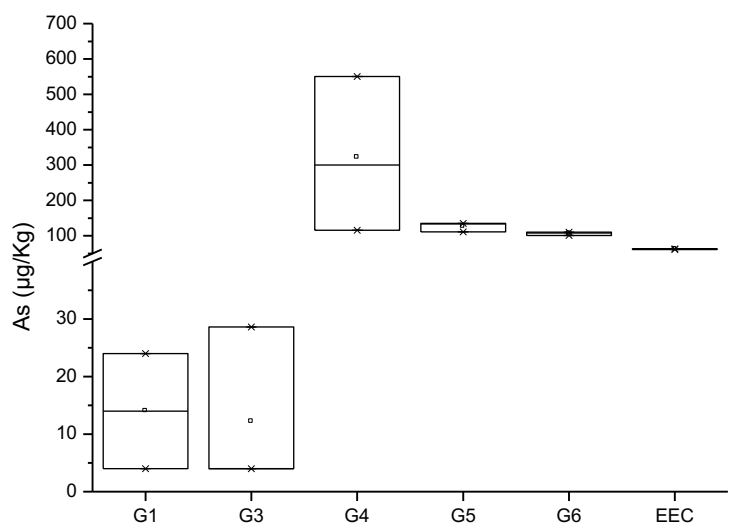

Figura 5. Distribución de arsénico total en granos de maíz obtenidos de las plantas de Camiña y de EEC

Algunos sectores presentaron concentraciones de arsénico inferiores al límite de detección (6 $\mu \mathrm{g} / \mathrm{kg}$ ), mientras que otros sectores se observó un amplio intervalo entre $30 \mu \mathrm{g} / \mathrm{kg}$ y el valor máximo en el sector 4 , con $550 \mu \mathrm{g} / \mathrm{kg}$. El valor promedio de arsénico en grano de Camiña fue de $140 \pm 100 \mu \mathrm{g} / \mathrm{kg}$, mientras que en la EEC se encontró un valor de $250 \pm 40 \mu \mathrm{g} / \mathrm{kg}$.

En Chile la normativa vigente del Decreto Supremo DS977-1996, Reglamento Sanitario de los Alimentos, (Diario Oficial de la República de Chile, Santiago, Chile, 13 de mayo de 1997), fija el nivel máximo de la concentración de arsénico en grano de $200 \mu \mathrm{g} / \mathrm{kg}$. En base a lo anterior, 
los granos de choclo de las plantas de maíz de la quebrada de Camiña se encuentran dentro de los valores permitidos por esta normativa, excepto la contramuestra G4B y la muestra del sector ECC que es levemente superior a la concentración sugerida por legislación. Además, se presentan concentraciones de arsénico normales al compararlos con los valores señalados para granos de maíz reportados en diversos estudios ${ }^{5}$. Las bajas concentraciones de arsénico, son debido a los procesos biológicos de la planta de bioacumulación y translocación, donde el fruto corresponde a la última sección de transporte de sustancias dentro de la morfología de las plantas 5 .

La tabla 3 muestra los PFT calculados desde la raíz hacia las hojas y desde la raíz hacia los granos, expresado en porcentaje. En este estudio, el valor medio de translocación de raíz a hojas fue de $3,17 \%$, con un intervalo entre 0,54 y $6,36 \%$, teniendo una amplia desviación estándar de 1,82; en otros trabajos se han reportado valores muy similares de $2,89 \%$ entre 0,9 y $6 \%$ y con una variación de 1,965. El PFT de la raíz hacia los granos de maíz no se logró determinar en el $37 \%$ de las muestras, debido a que el contenido de arsénico en las muestras de maíz en muchos fue inferior a LD. Una investigación similar en suelo, agua de riego y muestras de plantas de maíz, recolectadas de campos de maíz cerca de la zona minera de San Luis Potosí, México reporta de igual manera que la concentración de arsénico en los granos fue bajo el límite de detección4. En el caso del sector 3 se observaron PFT muy bajos en todas las muestras, esto concuerda con una translocación no significativa en las plantas de maíz a partir de raíces a los granos 5 .

Tabla 3. Valores de los FBC y PFT para los sectores en estudio

\begin{tabular}{llcc}
\hline & FBC & PFT hoja (\%) & PFT grano (\%) \\
\hline S1A & 3,27 & 3,96 & 0,00 \\
S1B & 4,77 & 2,98 & 0,02 \\
S1C & 2,99 & 5,90 & 0,02 \\
\hline S3A & 3,45 & 1,57 & 0,00 \\
S3B & 3,18 & 4,24 & 0,03 \\
S3C & 3,24 & 1,78 & 0,00 \\
\hline S4A & 1,29 & 3,69 & 0,00 \\
S4B & 1,32 & 2,64 & 0,28 \\
S4C & 1,65 & 2,96 & 0,06 \\
\hline S5A & 2,39 & 0,54 & 0,05 \\
S5B & 2,13 & 1,68 & 0,05 \\
S5C & 2,21 & 2,47 & 0,07 \\
\hline S6A & 1,05 & 3,91 & 0,10 \\
S6B & 0,90 & 3,37 & 0,11 \\
S6C & 1,51 & 3,76 & 0,08 \\
\hline EECA & 1,75 & 2,02 & 0,07 \\
EECB & 1,75 & 2,01 & 0,07 \\
EECC & 1,62 & 2,01 & 0,07
\end{tabular}




\section{CONCLUSIONES}

Las aguas naturales usadas para el riego de Zea mays L. cultivados en el valle de Camiña son dulces, levemente alcalinas, enriquecidas con nitrato y contaminadas con elevadas concentraciones de arsénico que superan en aproximadamente 40 veces la norma chilena para agua potable y cinco veces la norma de riego. Las aguas para riego del sector EEC cumplen con la norma nacional de riego. Los suelos cultivados de Camiña son arenosos, no salinos, de $\mathrm{pH}$ neutro-levemente alcalino, poseen escasa materia orgánica y están contaminados con boro y arsénico. Se tipificaron tres niveles, dos niveles elevados de arsénico 100 y $300 \mathrm{mg} / \mathrm{kg}$ y un nivel normal de $32 \mathrm{mg} / \mathrm{kg}$, que correlacionó positivo con COFO. Los suelos arenosos y levemente alcalinos y salinos del sector Canchones poseen una concentración del metaloide levemente más elevada de $50 \mathrm{mg} / \mathrm{kg}$.

Las plantas de maíz Zea mays L. bioconcentra arsénico con un factor de bioconcentración mayor que 1 , lo que indica un mecanismo de translocación del suelo a la raíz, resultando ser un excelente fitorremediador. El arsénico acumulado en planta tiende a almacenarse en la raíz, impidiendo concentraciones elevadas de arsénico en partes aéreas, a pesar de los altos niveles de arsénico en suelos. Las concentraciones de arsénico en las diferentes partes de la planta de maíz presentaron el siguiente orden: raíz $>$ hoja $>$ grano. El consumo de granos de choclo provenientes de Camiña no presenta riesgo, ya que presentaron concentraciones de arsénico relativamente bajas.

No existe una clara correlación entre la concentración de arsénico en agua y suelos. No se encontró relación entre el arsénico del suelo y en las matrices biológicas.

\section{AGRADECIMIENTOS}

Los autores agradecen el financiamiento a CONICYT-GORE Tarapacá -Universidad Arturo Prat, proyecto CONICYT REGIONAL/CIDERH R09I1001, CENIMA-UNAP y a las comunidades del valle de Camiña.

\section{REFERENCIAS BIBLIOGRÁFICAS}

1 Baig JA, Kazi TG, Shah AQ, Arain MB, Afridi HJ, Khan S, Khan S, Kandhro GA, Naeemullah, Soomro As. Evaluating the accumulation of arsenic in maize (Zea mays L.) plants from its growing media by cloud point extraction. Food Chem Toxicol. 2010; 48(11): 3051-3057.

2 Ali H, Khan E, Sajad MA. Phytoremediation of heavy metals-Concepts and applications. Chemosphere. 2013; 91: 869-881.

3. Marwa E, Meharg A, Rice CM. Risk assessment of potentially toxic elements in agricultural soils and maize tissues from selected districts in Tanzania. Sci Total Environ. 2012; 416: 180-186. 
4. Rosas JM, Guzmán JL, Alfaro JM, Hernández A, Perez LN, Hinojosa L. Evaluation of the transfer of soil arsenic to maize crops in suburban areas of San Luis Potosi, Mexico. Sci Total Environ. 2014; 497-498: 153-162.

5. Rosas JM, Guzmán JL, Hernández A, Garza MT, Hinojosa L. Arsenic accumulation in maize crop (Zea mays): A review. Sci Total Environ. 2014; 488-489: 176-187.

6. Bundschuh J, Litter M, Parvez F, Román-Ross G, Nicolli HB, Jean JS, et al. One century of arsenic exposure in Latin America: A review of history and occurrence from 14 countries. Sci Total Environ. 2012; 429:2 -35.

7. Muñoz O, Díaz OP, Leyton I, Núñez N, Devesa V, Súñer MA, Vélez D, Montoro R. Vegetables collected in the cultivated Andean area of northern Chile: total and inorganic arsenic contents in raw vegetables. J Agric Food Chem. 2002; 50(3):642-7.

8. Lictevout E, Mass C, Córdoba D, Herrera V, Payano R. Recursos Hídricos Región de Tarapacá: Diagnóstico y sistematización de la información. Chile: Centro de Investigación y Desarrollo en Recursos Hídricos (CIDERH). Iquique: Universidad Arturo Prat; 2013.

9. Astudillo F, Lacassie JP, Baeza L, Barrera J, Carrasco F, Castillo P, et al. Principales Características Geoquímicas de sedimentos de la Hoja Pisagua, Regiones de Tarapacá y de Arica y Parinacota, Chile. XVI Congreso Geológico Chileno; 4-8 Oct 2015; La Serena, Chile. Santiago de Chile: Sociedad Geológica de Chile; 2015. P 272-276.

10. APHA, AWWA \& WPCF. Métodos normalizados para el análisis del agua potable y residual. Madrid: Ediciones Díaz de Santos S.A.; 1992.

11. Sadzawka R, Carrasco M, Grez R, Mora M, Flores H, Neaman, A. Métodos de análisis recomendados para los suelos de Chile. Chile: Instituto de Investigaciones Agropecuarias, Serie actas INIA N³4, Santiago de Chile; 2006.

12. Herrera V. De Gregori I, Pinochet H. Assessment of trace elements and mobility of arsenic and manganese in lagoon sediments of the salars of Huasco and Coposa, Chilean Altiplano. J Chil Chem Soc. 2009; 54 (3): 281-288. 